\title{
Using Cauchy Distribution To Estimate Survival Function
}

\author{
Lecturer/ Hind Jawad Kadhim AlBderi \\ College of Administration and Economics / University of Al-Qadisiyah \\ hind.kadhum@qu.edu.iq \\ hind_satia@yahoo.com \\ http://orcid.org/0000-0003-1525
}

Article History: Received: 17 September 2020; Accepted: 04 October 2020; Published online: 5 April 2021

\begin{abstract}
This paper intends to estimate the unlabeled two parameters for Cauchy distribution model depend on employing the maximum likelihood estimator method to obtain the derivation of the point estimators for all unlabeled parameters depending on iterative techniques, as Newton - Raphson method, then to derive "Lindley approximation estimator method and then to derive Ordinary least squares estimator method. Applying all these methods to estimate related probability functions; death density function, cumulative distribution function, survival function and hazard function (rate function)".

"When examining the numerical results for probability survival function by employing mean squares error measure and mean absolute percentage measure, this may lead to work on the best method in modeling a set of real data".
\end{abstract}

\section{Introduction:}

The first publication of Cauchy distribution was in 1824 by the French mathematician Poisson; then it was only associated with the name Augustin Cauchy during an academic debate in 1853. Physicists call it the Lorentz distribution due to Hendrink Lorents.

Cauchy distribution is a continuous probability distribution of curves that has heavier tails than the normal distributions; and one of the most popular practical applications for this distribution is modeling the ratio of two normal random variables. $(1,2)$

"Introduce a tree-structured Bayesian network suitable for modelling directional data with bivariate wrapped Cauchy distributions. We describe the structure learning algorithm used to learn the Bayesian network". (2)

Because Cauchy distribution does not have limited moments greater than or equa to one and has no moment generating function, it is a stable distribution with a probability density function that can be explained analytically and therefore, Cauchy distribution might be considered as an example of a well-accepted results and conceple in statitics. (1)

This paper is divided as follows:- The objective of this paper, theoretical section, practical section, results and conclusions.

\section{The objective of this paper:}

This paper aims to examine sample type Breast cancer by using maximum likelihood estimator method (MLEM), Lindley approximation estimator method (LAEM) and Ordinary least squares estimator method ; and by comparing the three methods .

\section{Definition and properties:}

"The p.d.f for Cauchy distribution. Is":

$$
f_{p}(t ; \gamma, \delta)=\frac{\gamma}{\pi\left[\gamma^{2}+(t-\delta)^{2}\right]} \quad ; \quad t \geq 0 \ldots(1)
$$

$\Omega=\{(t ; \gamma, \delta) ; \gamma>0, \delta>0\}$

Where $\gamma$ : "is scale parameter"

$$
\delta \text { : "is location parameter" }
$$

"The cumulative distribution function for this distribution is": $F(t ; \gamma, \delta)=\frac{1}{2}+\frac{1}{\pi} \tan ^{-1}\left(\frac{t-\gamma}{\delta}\right) \ldots(2)$;

for $(-\infty<t<\infty ;-\infty<\gamma<\infty ; \delta>0)$ 
"It's survival function is given by": $s(t ; \gamma, \delta)=1-\left[\frac{1}{2}+\frac{1}{\pi} \tan ^{-1}\left(\frac{t-\gamma}{\delta}\right)\right] \ldots(3)$

"The hazard rate function is given by" : $\quad h(t ; \gamma, \delta)=\frac{\frac{\gamma}{\pi\left[\gamma^{2}+(t-\delta)^{2}\right]}}{1-\left[\frac{1}{2}+\frac{1}{\pi} \tan ^{-1}\left(\frac{t-\gamma}{\delta}\right)\right]}$
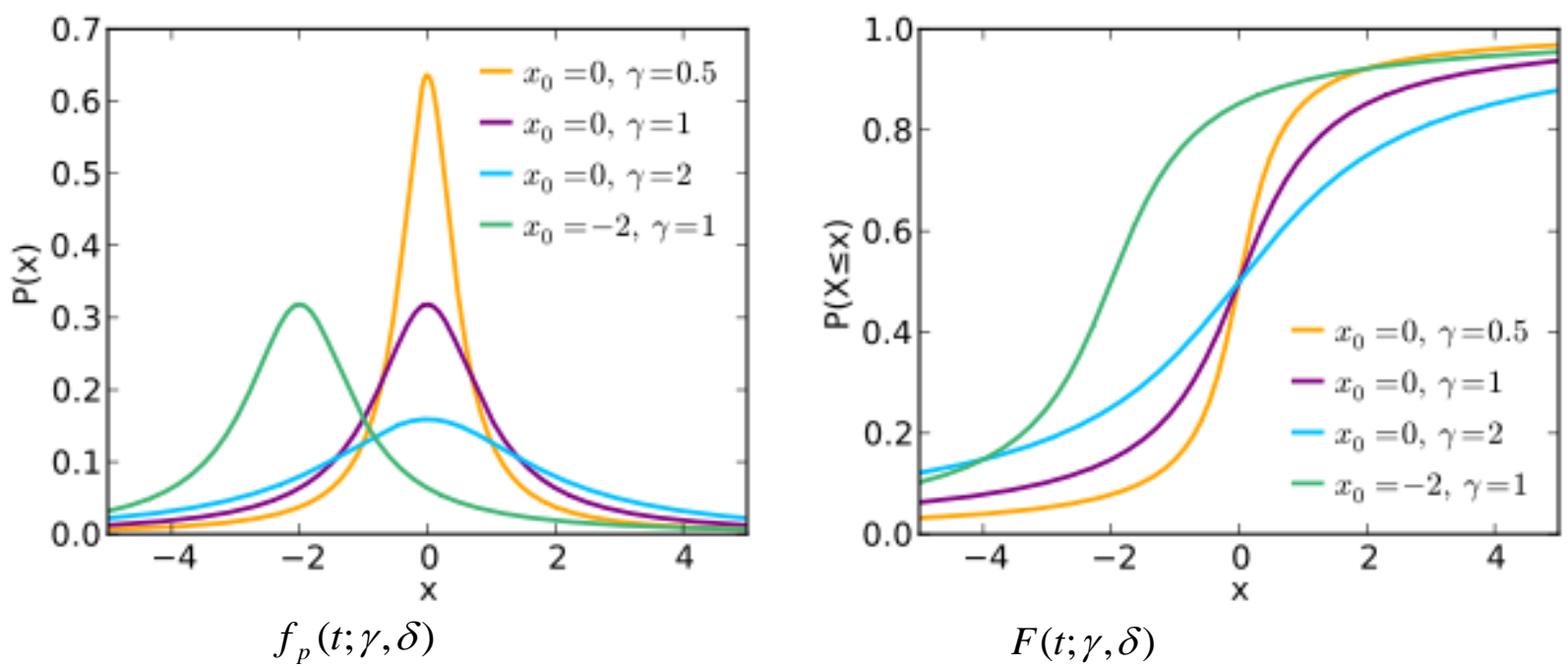

Maximum likelihood estimator method(MLEM):

"The MLM is the most common procedure to estimate the parameter $\phi$ which specifies a p.d.f. $f(t: \varphi)$, based on the observations $t_{1}, t_{2}, \ldots . ., t_{n}$ which were independent. sample from the distribution".

$$
\begin{aligned}
& L=\left[\prod_{i=1}^{n} f\left(t_{i} ; \gamma, \delta\right)\right]=\prod_{i=1}^{n}\left[\frac{\gamma}{\pi\left[\gamma^{2}+\left(t_{i}-\delta\right)^{2}\right]}\right] \ldots(5) \\
& \ln L=n \ln \gamma-n \ln \pi-\sum_{i=1}^{n} \ln \left[\gamma^{2}+\left(t_{i}-\delta\right)^{2}\right] \ldots(6) \\
& \frac{\partial \ln L}{\partial \gamma}=\frac{n}{\gamma}-\sum_{i=1}^{n} \frac{2 \gamma}{\gamma^{2}+\left(t_{i}-\delta\right)^{2}} \ldots(7) \quad ; \quad \frac{\partial \ln L}{\partial \gamma}=0 \quad ; \quad \frac{n}{\gamma}-\sum_{i=1}^{n} \frac{2 \gamma}{\gamma^{2}+\left(t_{i}-\delta\right)^{2}} \\
& \frac{\partial \ln L}{\partial \delta}=\sum_{i=1}^{n} \frac{2\left(t_{i}-\delta\right)}{\gamma^{2}+\left(t_{i}-\delta\right)^{2}} \ldots(9) \quad ; \quad \frac{\partial \ln L}{\partial \delta}=0 \quad ; \quad \sum_{i=1}^{n} \frac{2\left(t_{i}-\delta\right)}{\gamma^{2}+\left(t_{i}-\delta\right)^{2}} \ldots(10)
\end{aligned}
$$

"There is no chance to find the estimators for the parameters $(\gamma, \delta)$, and it is kind of difficulty to process the nonlinear equations thus, it is better to make use of iterative methods in numerical analysis as Newton-Raphson method which is the best way to get the estimate values and number of iteration".

"The Newton-Raphson method requires an initial value of each unknown parameters" $(\gamma, \delta)$.

This method follows :

$$
\begin{aligned}
& {\left[\begin{array}{l}
\gamma_{i+1} \\
\delta_{i+1}
\end{array}\right]=\left[\begin{array}{l}
\gamma_{i} \\
\delta_{i}
\end{array}\right]-J_{i}^{-1}\left[\begin{array}{l}
g_{1}(\gamma) \\
g_{2}(\delta)
\end{array}\right] \ldots} \\
& g_{1}(\gamma)=\frac{n}{\gamma}-\sum_{i=1}^{n} \frac{2 \gamma}{\gamma^{2}+\left(t_{i}-\delta\right)^{2}}
\end{aligned}
$$

$$
; \quad g_{2}(\delta)=\sum_{i=1}^{n} \frac{2\left(t_{i}-\delta\right)}{\gamma^{2}+\left(t_{i}-\delta\right)^{2}}
$$




$$
J_{i}^{-1}=\left[\begin{array}{ll}
\frac{\partial g_{1}(\gamma)}{\partial \gamma} & \frac{\partial g_{1}(\gamma)}{\partial \delta} \\
\frac{\partial g_{2}(\delta)}{\partial \gamma} & \frac{\partial g_{2}(\delta)}{\partial \delta}
\end{array}\right]
$$

$\frac{\partial g_{1}(\gamma)}{\partial \gamma}=-\frac{n}{\gamma^{2}}-\sum_{i=1}^{n} \frac{2\left(t_{i}-\delta\right)^{2}-2 \gamma^{2}}{\left[\gamma^{2}+\left(t_{i}-\delta\right)^{2}\right]^{2}}$

$\frac{\partial g_{2}(\delta)}{\partial \gamma}=-\sum_{i=1}^{n} \frac{4 \gamma\left(t_{i}-\delta\right)}{\left[\gamma^{2}+\left(t_{i}-\delta\right)^{2}\right]^{2}}$

$$
\left[\begin{array}{c}
\varepsilon_{i+1}(\gamma) \\
\varepsilon_{i+1}(\delta)
\end{array}\right]=\left\lfloor\left[\begin{array}{l}
\gamma_{i+1} \\
\delta_{i+1}
\end{array}\right]-\left[\begin{array}{l}
\gamma_{i} \\
\delta_{i}
\end{array}\right]\right.
$$

\section{"Lindley approximation estimator method (LAEM)":}

"Lindley procedure was presented in (1980) first time to approximate the ratio of the integrals of the form(3):

$$
\frac{\int w(\lambda) e^{L(\lambda)} d \lambda}{\int v(\lambda) e^{L(\lambda)} d \lambda} \ldots(20)
$$

"Where $\lambda=\left(\lambda_{1}, \lambda_{2}, \ldots . ., \lambda_{n}\right)$ are parameters, $L(\lambda)$ is the logarithm of the likelihood function, $w(\lambda)$ and $v(\lambda)$ are any random functions for parameters".

Let $v(\lambda)$ be the prior distribution of $\lambda$ and $w(\lambda)=u(\lambda) v(\lambda)$. From (20) we can get posterior expectation which is as follow :

$$
E[u(\lambda) \mid \underline{x}]=\frac{\int u(\lambda) e^{L(\lambda)+P(\lambda)} d \lambda}{\int e^{L(\lambda)+P(\lambda)} d \lambda}
$$

Where $P(\lambda)=\log [v(\lambda)]$

$$
\begin{aligned}
& \hat{R}=R(\hat{\gamma} \hat{\delta})+\frac{1}{2}\left[B+l_{30} C_{12}+l_{03} C_{21}+l_{21} D_{12}+l_{12} D_{21}\right]+p_{1} B_{12}+p_{2} B_{12} \ldots(22) \\
& B=\sum_{i=1}^{2} \sum_{j=1}^{2} Q_{i j} T_{i j} \quad ; \quad i, j=1,2 \ldots \ldots \ldots \ldots(23) \quad ; \quad l_{i j}=\frac{\partial^{i+j} L(\gamma, \delta)}{\partial \gamma^{i} \partial \delta^{j}} \ldots(24) \\
& p_{i}=\frac{\partial p}{\partial \delta_{i}} \quad, \quad p=\ln \Pi(\gamma, \delta) \ldots(25) \\
& Q_{1}=\frac{\partial R}{\partial \gamma} \quad, \quad Q_{2}=\frac{\partial R}{\partial \delta} \quad, \quad Q_{i j}=\frac{\partial^{2} R}{\partial \gamma_{i} \partial \delta_{j}} \ldots(26) \\
& B_{i j}=Q_{i} T_{i j}+Q_{j} T_{j i} \ldots(27) \quad ; \quad C_{i j}=\left(Q_{i} T_{i j}+Q_{j} T_{i j}\right) T_{i i} \ldots(28) \quad ; \quad D_{i j}=3 Q_{i} T_{i i} T_{i j}+Q_{i}\left(T_{i i} T_{j j}+2 T_{i j}^{2}\right)
\end{aligned}
$$


$f_{p}(t ; \gamma, \delta)=\frac{\gamma}{\pi\left[\gamma^{2}+(t-\delta)^{2}\right]}$

We assumed that $\gamma, \delta$ have the following Gamma conjugate prior distribution such that $: \gamma \sim \sqrt{(n, a)} ; \delta \sim \sqrt{(n, b)}$

$f(\gamma)= \begin{cases}\frac{a^{n}}{\sqrt{(n)}} \gamma^{n-1} e^{-a \gamma} & a>0, n>0, \gamma>0 \\ 0 & \text { o.w }\end{cases}$
$f(\delta)= \begin{cases}\frac{b^{n}}{\sqrt{(n)}} \delta^{n-1} e^{-b \delta} & b>0, n>0, \delta>0 \\ 0 & \text { o.w }\end{cases}$

$p\left(\gamma, \delta \mid t_{1}, t_{2}, \ldots, t_{n}\right)=\frac{L\left(t_{1}, t_{2}, \ldots, t_{n} ; \gamma, \delta\right) f(\gamma) f(\delta)}{\int_{0}^{\infty} \int_{0}^{\infty} L\left(t_{1}, t_{2}, \ldots, t_{n} ; \gamma, \delta\right) f(\gamma) f(\delta) d \gamma d \delta}$

$\hat{R}=E[R(\gamma, \delta)]=\frac{\int_{0}^{\infty} \int_{0}^{\infty} R(\gamma, \delta) L\left(t_{1}, t_{2}, \ldots, t_{n} ; \gamma, \delta\right) f(\gamma) f(\delta) d \gamma d \delta}{\int_{0}^{\infty} \int_{0}^{\infty} L\left(t_{1}, t_{2}, \ldots, t_{n} ; \gamma, \delta\right) f(\gamma) f(\delta) d \gamma d \delta}$

$\hat{R}=\frac{\int_{0}^{\infty} \int_{0}^{\infty} R(\gamma, \delta){ }_{i=1}^{n}\left[\frac{\gamma}{\pi\left[\gamma^{2}+\left(t_{i}-\delta\right)^{2}\right]}\right] \frac{a^{n}}{\sqrt{(n)}} \gamma^{n-1} e^{-a \gamma} \frac{b^{n}}{\sqrt{(n)}} \delta^{n-1} e^{-b \delta} d \gamma d \delta}{\int_{0}^{\infty} \int_{0}^{\infty} \pi\left[\frac{\gamma}{\pi\left[\gamma^{2}+\left(t_{i}-\delta\right)^{2}\right]}\right] \frac{a^{n}}{\sqrt{(n)}} \gamma^{n-1} e^{-a \gamma} \frac{b^{n}}{\sqrt{(n)}} \delta^{n-1} e^{-b \delta} d \gamma d \delta}$

“We make used of Lindley's approximate $\hat{R}$ which approximate the ratio of the two integrals to obtain Bayes estimators approximation that can be resulted as follows" :

Using equation (6) we get the following:

$$
\begin{aligned}
& l_{12}=\frac{\partial^{3} \ln L(\gamma, \delta)}{\partial \gamma \partial \delta^{2}}=\sum_{i=1}^{n} \frac{4 \gamma\left[\gamma^{2}+\left(t_{i}-\delta\right)^{2}(1-4 \gamma)\right]}{\left[\gamma^{2}+\left(t_{i}-\delta\right)^{2}\right]^{3}} \ldots \\
& l_{21}=\frac{\partial^{3} \ln L(\gamma, \delta)}{\partial \gamma^{2} \partial \delta}=\sum_{i=1}^{n} \frac{4\left(t_{i}-\delta\right)\left[3 \gamma^{2}-\left(t_{i}-\delta\right)^{2}\right]}{\left[\gamma^{2}+\left(t_{i}-\delta\right)^{2}\right]^{3}} \ldots \\
& l_{03}=\frac{\partial^{3} \ln L(\gamma, \delta)}{\partial \delta^{3}}=\sum_{i=1}^{n} \frac{4\left(t_{i}-\delta\right)\left[\left(t_{i}-\delta\right)^{2}-3 \gamma^{2}\right]}{\left[\gamma^{2}+\left(t_{i}-\delta\right)^{2}\right]^{3}} \ldots \\
& l_{30}=\frac{\partial^{3} \ln L(\gamma, \delta)}{\partial \gamma^{3}}=\frac{2 n}{\gamma^{3}}+\sum_{i=1}^{n} \frac{4 \gamma\left[3\left(t_{i}-\delta\right)^{2}-\gamma^{2}\right]}{\left[\gamma^{2}+\left(t_{i}-\delta\right)^{2}\right]^{3}} \ldots
\end{aligned}
$$


When $R(\gamma, \delta)=\gamma \quad ; \quad Q_{1}=\frac{\partial R}{\partial \gamma}=1 \quad, \quad Q_{2}=\frac{\partial R}{\partial \delta}=0 \quad, \quad Q_{12}=Q_{21}=\frac{\partial^{2} R}{\partial \gamma_{i} \partial \delta_{j}}=0$

$E=\frac{\partial^{2} \ln L(\gamma, \delta)}{\partial \gamma^{2}}=-\frac{n}{\lambda^{2}}-\sum_{i=1}^{n} \frac{2\left(t_{i}-\delta\right)^{2}-2 \gamma^{2}}{\left[\gamma^{2}+\left(t_{i}-\delta\right)^{2}\right]^{2}}$

$F=\frac{\partial^{2} \ln L(\gamma, \delta)}{\partial \delta^{2}}=\sum_{i=1}^{n} \frac{2\left(t_{i}-\delta\right)^{2}-2 \gamma^{2}}{\left[\gamma^{2}+\left(t_{i}-\delta\right)^{2}\right]^{2}}$

$G=\frac{\partial^{2} \ln L(\gamma, \delta)}{\partial \delta \partial \gamma}=\frac{\partial^{2} \ln L(\gamma, \delta)}{\partial \gamma \partial \delta}=-\sum_{i=1}^{n} \frac{4 \gamma\left(t_{i}-\delta\right)}{\left[\gamma^{2}+\left(t_{i}-\delta\right)^{2}\right]^{2}}$

$T_{11}=\frac{F}{E F-G^{2}} \ldots(43) \quad ; \quad T_{22}=\frac{E}{E F-G^{2}}=\ldots(44) \quad ; \quad T_{12}=T_{21}=\frac{-G}{E F-G^{2}} \ldots$

$p=\ln f(\gamma, \delta)=n \ln a-\ln \sqrt{(n)}+(n-1) \ln \gamma-a \gamma+n \ln \delta-\ln \sqrt{(n)}+(n-1) \ln \delta-b \delta$

$p_{1}=\frac{\partial p}{\partial \gamma}=\frac{n-1}{\gamma}-a \ldots(47) \quad ; \quad p_{2}=\frac{\partial p}{\partial \delta}=\frac{n-1}{\delta}-b$

$B=\sum_{i=1}^{2} \sum_{j=1}^{2} Q_{i j} T_{i j}=Q_{12} T_{12}+Q_{21} T_{21}=0$

$C_{12}=T_{12} T_{11} \quad ; \quad C_{21}=T_{21} T_{22} \ldots(50)$

$D_{12}=3 T_{11} T_{12}+T_{11} T_{22}+2 T_{12}^{2} \quad ; \quad D_{21}=0$

$B_{12}=T_{12} \quad ; \quad B_{21}=T_{21}$

$\hat{\gamma}=\gamma_{M L E}^{\wedge}+\frac{1}{2}\left[l_{30} T_{12} T_{11}+l_{03} T_{21} T_{22}+l_{21}\left(3 T_{11} T_{12}+T_{11} T_{22}+2 T_{12}^{2}\right)\right]+\left(\frac{n-1}{\gamma}-a\right) T_{12}+\left(\frac{n-1}{\delta}-b\right) T_{12}$

When $R(\gamma, \delta)=\delta \Rightarrow Q_{1}=\frac{\partial R}{\partial \gamma}=0 \quad, \quad Q_{2}=\frac{\partial R}{\partial \delta}=1 \quad, \quad Q_{12}=Q_{21}=\frac{\partial^{2} R}{\partial \gamma_{i} \partial \delta_{j}}=0$

From equation (49), (50) and $\quad D_{12}=0 \quad ; \quad D_{21}=3 T_{22} T_{21}+T_{22} T_{11}+2 T_{2}^{2}$

$B_{12}=T_{21} \quad ; \quad B_{21}=T_{21} \ldots(56)$

$\hat{\delta}=\delta_{M L E}^{\wedge}+\frac{1}{2}\left[l_{30} T_{12} T_{11}+l_{03} T_{21} T_{22}+l_{12}\left(3 T_{22} T_{21}+T_{22} T_{11}+2 T_{21}^{2}\right)\right]+\left(\frac{n-1}{\gamma}-a\right) T_{21}+\left(\frac{n-1}{\delta}-b\right) T_{21}$

Ordinary Least Squares Estimator Method (OLSEM):

"The OLSEM is the most used way to estimate parameters in linear or nonlinear model. Researchers make use of this method to lessen the sum squares differences concerning observed sample values and expected estimated values by linear approximation". $(4,5)$

$Y=\beta_{0}+\beta_{1} x+\varepsilon$
$\sum_{i=1}^{n} \varepsilon_{i}^{2}=\sum_{i=1}^{n}\left[y_{i}-\hat{y}_{i}\right]^{2}$

$\begin{array}{ll}; & \varepsilon=Y_{i}-\hat{\beta}_{0}-\hat{\beta}_{1} x_{i} \ldots(59) \\ ; & \sum_{i=1}^{n} \varepsilon_{i}^{2}=\sum_{i=1}^{n}\left[y_{i}-\hat{\beta}_{0}-\hat{\beta}_{1} x_{i}\right]^{2}\end{array}$ 


$$
\begin{aligned}
& F\left(t_{i}\right)=\frac{1}{2}+\frac{1}{\pi} \tan ^{-1}\left(\frac{t_{i}-\gamma}{\delta}\right) \ldots(62) \quad ; \quad \varepsilon=F\left(t_{i}\right)-\frac{1}{2}-\frac{1}{\pi} \tan ^{-1}\left(\frac{t_{i}-\gamma}{\delta}\right) \\
& \sum_{i=1}^{n} \varepsilon_{i}^{2}=\sum_{i=1}^{n}\left[F\left(t_{i}\right)-\frac{1}{2}-\frac{1}{\pi} \tan ^{-1}\left(\frac{t_{i}-\gamma}{\delta}\right)\right] \ldots(64) \quad \text { Let } \sum_{i=1}^{n} \varepsilon_{i}^{2}=H(\gamma, \delta) \\
& H(\gamma, \delta)=\sum_{i=1}^{n}\left[F\left(t_{i}\right)-\frac{1}{2}-\frac{1}{\pi} \tan ^{-1}\left(\frac{t_{i}-\gamma}{\delta}\right)\right] \ldots \\
& \frac{\partial H}{\partial \gamma}=-\frac{1}{\pi} \sum_{i=1}^{n}\left(\frac{\frac{-1}{\delta}}{1+\left(\frac{t_{i}-\gamma}{\delta}\right)^{2}}\right) \ldots(66) \quad ; \quad \frac{\partial H}{\partial \gamma}=0 \quad ; \quad-\frac{1}{\pi} \sum_{i=1}^{n}\left(\frac{\frac{-1}{\delta}}{1+\left(\frac{t_{i}-\gamma}{\delta}\right)^{2}}\right) \\
& \frac{\partial H}{\partial \delta}=-\frac{1}{\pi} \sum_{i=1}^{n}\left(\frac{\left.\frac{-1\left(t_{i}-\gamma\right)}{\delta^{2}}\right)}{1+\left(\frac{t_{i}-\gamma}{\delta}\right)^{2}}\right) .(68) \quad ; \quad \frac{\partial H}{\partial \delta}=0 \quad ; \quad-\frac{1}{\pi} \sum_{i=1}^{n}\left(\frac{\frac{-1\left(t_{i}-\gamma\right)}{\delta^{2}}}{1+\left(\frac{t_{i}-\gamma}{\delta}\right)^{2}}\right)
\end{aligned}
$$

There is no chance to find the estimators for the parameters $(\gamma, \delta)$, and it is kind of difficulty to process the nonlinear equations thus, it is better to make use of iterative methods in numerical analysis as Newton-Raphson method which is the best way to get the estimate values and number of iteration.

The Newton-Raphson method requires an initial value of each unknown parameters $(\gamma, \delta)$.

$$
\begin{aligned}
& {\left[\begin{array}{l}
\gamma_{i+1} \\
\delta_{i+1}
\end{array}\right]=\left[\begin{array}{l}
\gamma_{i} \\
\delta_{i}
\end{array}\right]-J_{i}^{-1}\left[\begin{array}{l}
z_{1}(\gamma) \\
z_{2}(\delta)
\end{array}\right] \ldots(70)} \\
& z_{1}(\gamma)=-\frac{1}{\pi} \sum_{i=1}^{n}\left(\frac{\frac{-1}{\delta}}{1+\left(\frac{t_{i}-\gamma}{\delta}\right)^{2}}\right) \ldots(71) \quad ; \quad z_{2}(\delta)=-\frac{1}{\pi} \sum_{i=1}^{n}\left(\frac{\frac{-1\left(t_{i}-\gamma\right)}{\delta^{2}}}{1+\left(\frac{t_{i}-\gamma}{\delta}\right)^{2}}\right) \\
& J_{i}^{-1}=\left[\begin{array}{cc}
\frac{\partial z_{1}(\gamma)}{\partial \gamma} & \frac{\partial z_{1}(\gamma)}{\partial \delta} \\
\frac{\partial z_{2}(\delta)}{\partial \gamma} & \frac{\partial z_{2}(\delta)}{\partial \delta}
\end{array}\right] \\
& \frac{\partial z_{1}(\gamma)}{\partial \gamma}=\frac{1}{\pi} \sum_{i=1}^{n}\left(\frac{\frac{2\left(t_{i}-\lambda\right)}{\delta^{3}}}{\left[1+\left(\frac{t_{i}-\gamma}{\delta}\right)^{2}\right]^{2}}\right) \ldots(74) \quad ; \quad \frac{\partial z_{1}(\gamma)}{\partial \delta}=-\frac{1}{\pi} \sum_{i=1}^{n}\left(\frac{\frac{1}{\delta^{2}}\left[1-\left(\frac{t_{i}-\lambda}{\delta}\right)^{2}\right.}{\left[1+\left(\frac{t_{i}-\gamma}{\delta}\right)^{2}\right]^{2}}\right) \\
& \frac{\partial z_{2}(\delta)}{\partial \gamma}=-\frac{1}{\pi} \sum_{i=1}^{n}\left(\frac{\frac{1}{\delta^{2}}\left[1-\left(\frac{t_{i}-\lambda}{\delta}\right)^{2}\right.}{\left[1+\left(\frac{t_{i}-\gamma}{\delta}\right)^{2}\right]^{2}}\right) \ldots(76) \quad ; \quad \frac{\partial z_{2}(\delta)}{\partial \delta}=-\frac{1}{\pi} \sum_{i=1}^{n}\left(\frac{\frac{2\left(t_{i}-\lambda\right)}{\delta^{3}}}{\left[1+\left(\frac{t_{i}-\gamma}{\delta}\right)^{2}\right]^{2}}\right)
\end{aligned}
$$


$\left[\begin{array}{c}\varepsilon_{i+1}(\gamma) \\ \varepsilon_{i+1}(\delta)\end{array}\right]=\left|\left[\begin{array}{l}\gamma_{i+1} \\ \delta_{i+1}\end{array}\right]-\left[\begin{array}{l}\gamma_{i} \\ \delta_{i}\end{array}\right]\right| \ldots$

\section{Results and Discussion:}

The Educational Hospital in Diwaniyah province was the place from which the data was gathered.

Keeping in mind that this work relies on data taken from real life, it is reached to select this kind of cancer (Breast cancer) because it is remarkable widespread and deadly in Iraq; this disease has failure time (death time) which is phenomenon in this paper.

The study of this paper covers a period of six months; it begins from Jun 2019 until December 2019; it is an experiment that includes (14) patients.(12) patients were dead and (2) patients remain alive .

When applying the test statistic (Kolmogorov-Smirnov) depending upon statistical programming (EasyFit 5.5 Professional) in order to fit Cauchy distribution data, it is discovered that the calculated value is (0. 11034), this means data is distributed according to Cauchy distribution .

The null and alternative hypotheses are as follows :

$H_{0}$ : The survival time data is distributed as Cauchy.

$H_{1}$ : The survival time data is not distributed as Cauchy.

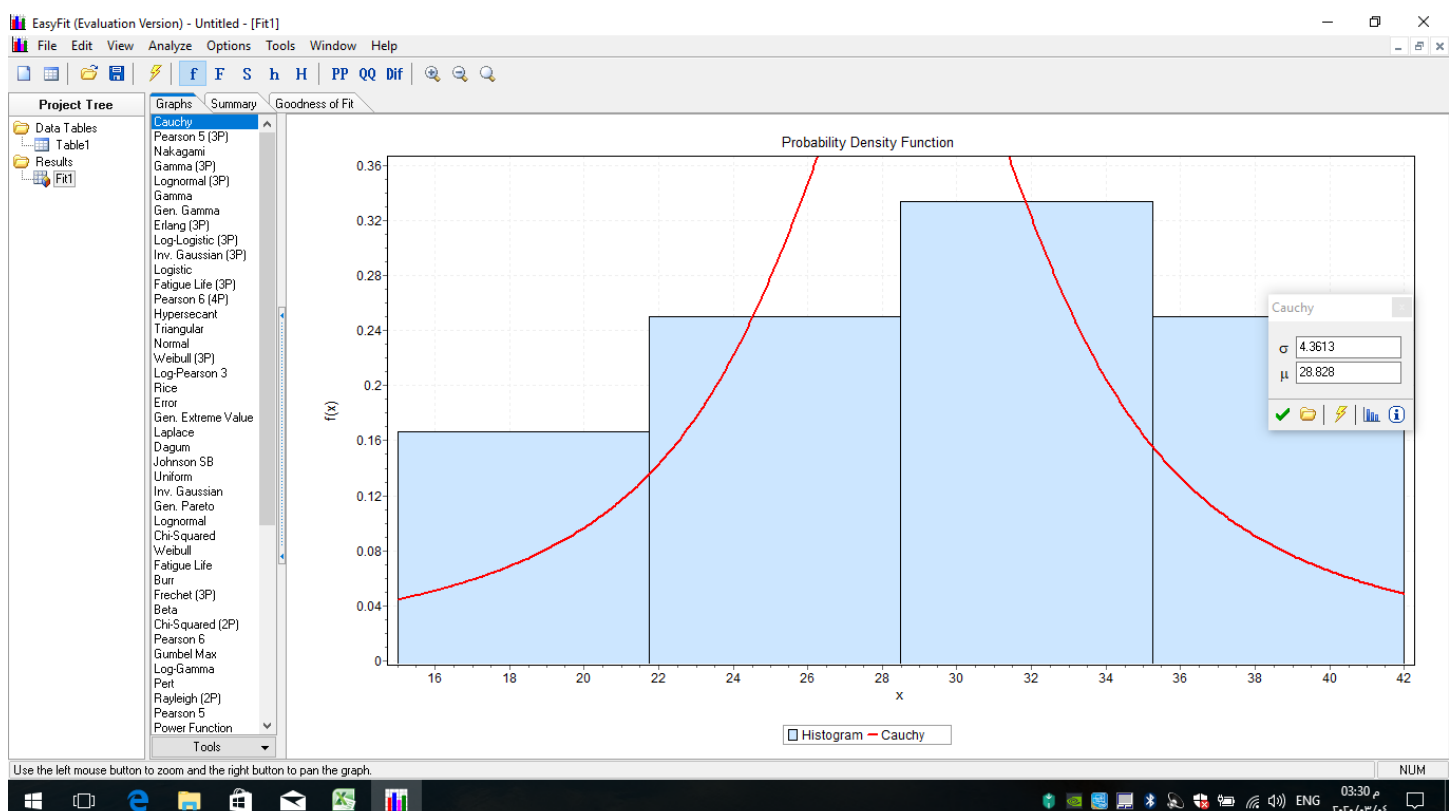

Fit the data for Breast cancer from Educational Hospital Diwaniyah 
Lecturer/ Hind Jawad Kadhim AlBderi

1 Easyfit (Evaluation Version) - Untitled - [Fitt]

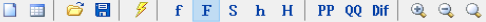

Project Tree Giaphs Summary Goodness of fit

Graphs Summary Goodness
Couchy
Pearson $5[3 P]$

Nakkagani
Gamma $3 P$

Gammana
Lognomal
Ganma
Gen Gen Gam

Gen. Gamma
Erlang ( $3 P]$

Log-Logistic (3P])
Inv. Gaussian (3P]

Logistic
Fatigu Lifi (3P)
Pearson 6 (4P)

Hearson 6 cant
Typersecant
Triangular

Triangular
Normal
Weibull (3P)
Log.Pearson 3

Log.Pear
Rice
Evar

Error
Gien. Extreme Value

Gen. Extr
Loplace
Daoum
Juhnson

Daoum
Johnson SB
Unitiom

Unifiom
Inv Gaussian

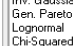

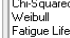

Fatigue Lite
Eur
Firchet 3 (3P)

Frechet [3P]
Beta

Reta
Chisquared $(2 P)$
Pearson 6

Gunbel Ma:

Pert
Rayleigh (2P)
Pearson 5

Cumulative Distribution Function

Tools

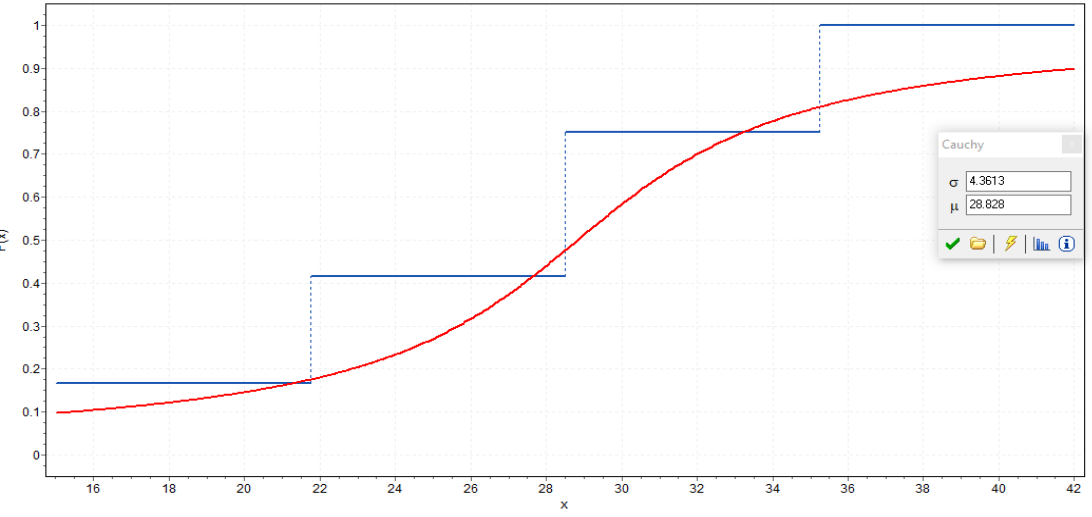

Use the left mouse button to zoorm and the right bullonto

- Sample - Cauchy

II Easyfit (Evaluation Version) - Untitled - [Fit1]

Wile Edit View Analyze Options Tools Window Help

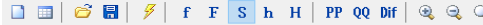

Project Tree Giaphs Summaly Goodness of Fit
Dota Tables

Data Tables

- Tables

\begin{tabular}{|l|l|l}
\hline $\begin{array}{l}\text { Peacrson } 5[3 P] \\
\text { Nakagani }\end{array}$ & Survival Function \\
\hline
\end{tabular}

Gamma [3P]
Lognormal (3)

Gamma
Gent. Gamma
Erlang (3P)

Log-Logistic (3P]
Inv. Gaussian (3P)

Logistic
Fatigue Lite (3P)
Pearson 6 (4P)

Heapersecant
Triangular

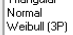

Error Gen. Extreme Value

Leplace
Lagum
Dagum

Uniniorm

Inv. Gaussian
Gen. Pareto
Lognormal

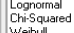

Weibull
Fatigue Life
Burr

Ferechet (3P)
Betat

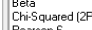

Pearson 6
Gumbel 6 a
Lumb

Loumb-Giamm
Pett

Rayleigh (2P)

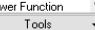

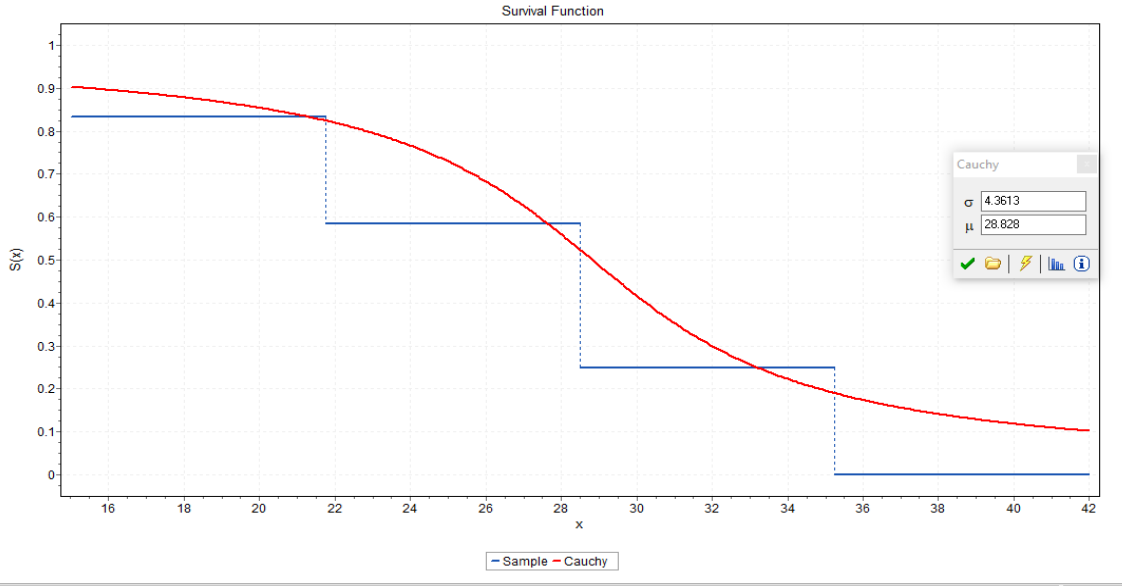

Wi Easyfit (Evaluation Version) - Untitled - [Fit1]

File Edit View Analyze Options Tools Window Hep

- 口 $\times$

口

Project Tree Giaphs Summarly Goodness of fit

Data Tables

$\square$ Resuls

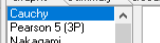

Gammana (3P)

Lognormal ( $3 P$ )
Gamma

Gen. Gamma
Erlang (3P)

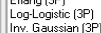

Logistic
Fatiug Life (3P)

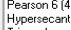

Triangula
Nomal
Normal

Normal
Weibul (3P)
Log-Pearson 3

Error
Gen Extreme Value
Laplace

Laplace
Dagum
Juhnson SB

Johnson SB

Inv. Gaussiat
Gen. Pareto
Lognormal

Lognorma
Chis Sual
Weibull

Weibull
Fatique Life
Bur

Bur
Frechet (3P)

Beta (IP)
Chisquared (2P)

\begin{tabular}{|l} 
Chi-Gquared \\
Pearson 6 \\
Gumbel Wax \\
\hline
\end{tabular}

Log-Garnm

Fayleigh (2P)
Pearson 5
Power Function

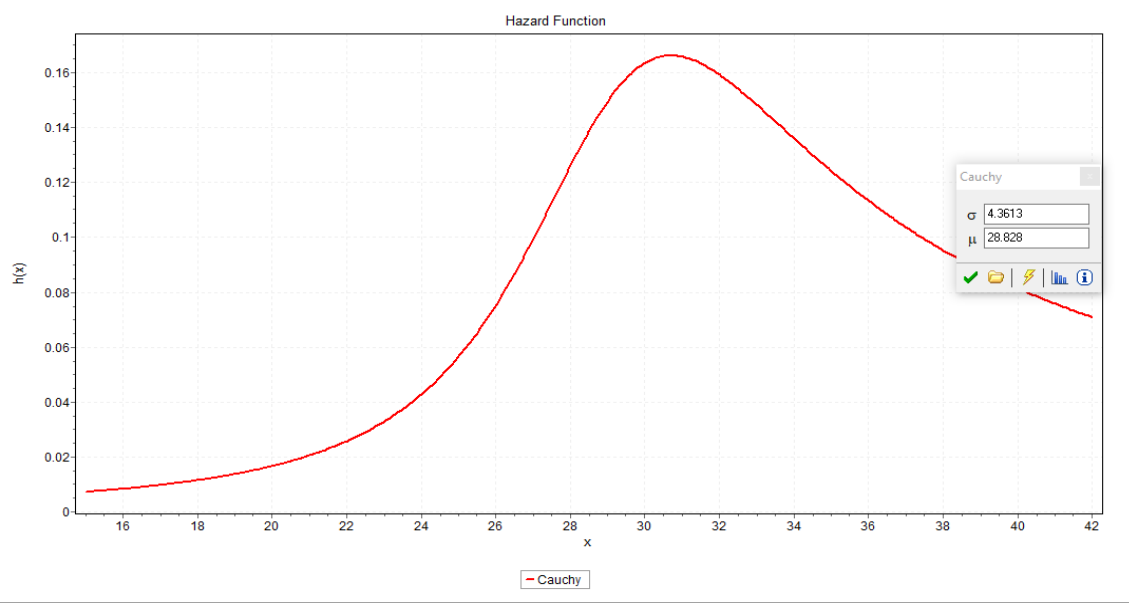


When applying MATLAB (R2014a), the estimated parameters results are as follows :

The assumed initial values for two-parameters are as follows:

Table (1)

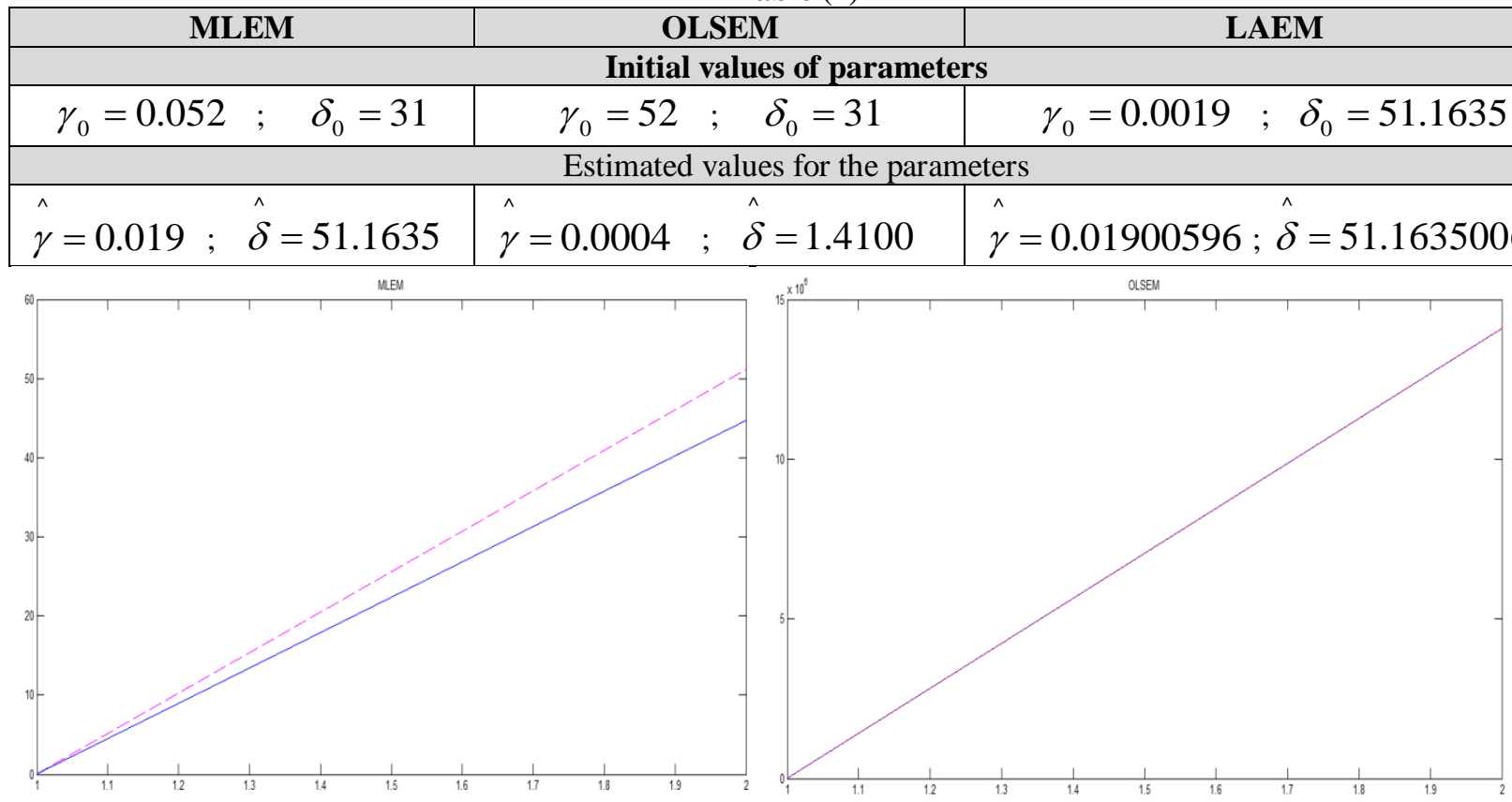

After that , using these estimated values for two-parameters in Cauchy distribution to find the numerical values for $f(t), F(t), s(t)$ and $h(t)$.

Table (2) : Estimated values for functions $f(t), F(t), s(t), h(t)$ by LAEM

\begin{tabular}{|c|c|c|c|c|}
\hline Failure Time & $f(t)$ & $F(t)$ & $s(t)$ & $h(t)$ \\
\hline 15 & $4.62 \mathrm{E}-07$ & 5.698456 & 0.499992 & $9.25 \mathrm{E}-07$ \\
\hline 21 & $6.65 \mathrm{E}-07$ & 7.599852 & 0.499988 & $1.33 \mathrm{E}-06$ \\
\hline 23 & $7.62 \mathrm{E}-07$ & 8.201278 & 0.499986 & $1.52 \mathrm{E}-06$ \\
\hline 25 & $8.83 \mathrm{E}-07$ & 8.785386 & 0.499984 & $1.77 \mathrm{E}-06$ \\
\hline 28 & $1.13 \mathrm{E}-06$ & 9.628227 & 0.499979 & $2.25 \mathrm{E}-06$ \\
\hline 29 & $1.23 \mathrm{E}-06$ & 9.900173 & 0.499978 & $2.46 \mathrm{E}-06$ \\
\hline 29 & $1.23 \mathrm{E}-06$ & 9.900173 & 0.499978 & $2.46 \mathrm{E}-06$ \\
\hline 30 & $1.35 \mathrm{E}-06$ & 10.1676 & 0.499975 & $2.70 \mathrm{E}-06$ \\
\hline 34 & $2.05 \mathrm{E}-06$ & 11.19218 & 0.499963 & $4.11 \mathrm{E}-06$ \\
\hline 38 & $3.49 \mathrm{E}-06$ & 12.14562 & 0.499936 & $6.98 \mathrm{E}-06$ \\
\hline 39 & $4.09 \mathrm{E}-06$ & 12.37313 & 0.499925 & $8.18 \mathrm{E}-06$ \\
\hline 42 & $7.20 \mathrm{E}-06$ & 13.03039 & 0.499869 & $1.44 \mathrm{E}-05$ \\
\hline
\end{tabular}

$\left.\operatorname{MSE}\left[\hat{s}\left(t_{i}\right)\right]=\frac{1}{n} \sum_{i=1}^{n} \hat{[s}\left(t_{i}\right)-s\left(t_{i}\right)\right]^{2}=0.014458476$

$\operatorname{MAPE}\left[\hat{s}\left(t_{i}\right)\right]=\frac{1}{n} \sum_{i=1}^{n}\left|\frac{\hat{s}\left(t_{i}\right)-s\left(t_{i}\right)}{s\left(t_{i}\right)}\right|=0.458202743$ 
Lecturer/ Hind Jawad Kadhim AlBderi

Table (3) : Estimated values for functions $f(t), F(t), s(t), h(t)$ by OLSEM

\begin{tabular}{|c|c|c|c|c|}
\hline Failure Time & $f(t)$ & $F(t)$ & $s(t)$ & $h(t)$ \\
\hline 15 & $7.72 \mathrm{E}-08$ & 28.52456448 & 0.499998592 & $1.55 \mathrm{E}-07$ \\
\hline 21 & $9.01 \mathrm{E}-08$ & 28.47754203 & 0.499998358 & $1.80 \mathrm{E}-07$ \\
\hline 23 & $9.51 \mathrm{E}-08$ & 28.46022013 & 0.499998267 & $1.90 \mathrm{E}-07$ \\
\hline 25 & $1.20 \mathrm{E}-07$ & 28.38075918 & 0.499997815 & $2.40 \mathrm{E}-07$ \\
\hline 28 & $1.56 \mathrm{E}-07$ & 28.28014998 & 0.499997161 & $3.11 \mathrm{E}-07$ \\
\hline 29 & $1.67 \mathrm{E}-07$ & 28.25067063 & 0.499996952 & $3.34 \mathrm{E}-07$ \\
\hline 29 & $1.67 \mathrm{E}-07$ & 28.25067063 & 0.499996952 & $3.34 \mathrm{E}-07$ \\
\hline 30 & $1.80 \mathrm{E}-07$ & 28.21909072 & 0.499996718 & $3.60 \mathrm{E}-07$ \\
\hline 34 & $2.29 \mathrm{E}-07$ & 28.10923657 & 0.499995831 & $4.57 \mathrm{E}-07$ \\
\hline 38 & $2.73 \mathrm{E}-07$ & 28.0201338 & 0.499995022 & $5.46 \mathrm{E}-07$ \\
\hline 39 & $3.32 \mathrm{E}-07$ & 27.91412867 & 0.499993954 & $6.63 \mathrm{E}-07$ \\
\hline 42 & $6.89 \mathrm{E}-07$ & 27.42767438 & 0.499987437 & $1.38 \mathrm{E}-06$ \\
\hline
\end{tabular}

$$
\begin{aligned}
& \left.\operatorname{MSE}\left[\hat{s}\left(t_{i}\right)\right]=\frac{1}{n} \sum_{i=1}^{n} \hat{[s}\left(t_{i}\right)-s\left(t_{i}\right)\right]^{2}=0.014467495 \\
& \left.\operatorname{MAPE} \hat{s}\left(t_{i}\right)\right]=\frac{1}{n} \sum_{i=1}^{n}\left|\frac{\hat{s}\left(t_{i}\right)-s\left(t_{i}\right)}{s\left(t_{i}\right)}\right|=0.0458332972
\end{aligned}
$$

\section{Conclusions:}

1- We notice in both methods that the estimated values of the probability survival function decrease with increasing failure times (an inverse relationship between them).

2- We notice in both methods that the estimated values of the potential risk function increase with increasing times of failure (a direct relationship between them).

3- It is recommended to use (LAEM) of Cauchy distribution of Breast cancer by employing MSE criterion.

\section{References:}

(1) Gerald Haas , Lee Bain and Gharles Antle 1970 "Inferences for the Cauchy distribution based on maximum likelihood estimators". Printed in Great Britain. Biometrika , 57, 2 , PP.403.

(2) M. H. Tahir , M. Zubair, Gauss M, Cordeiro, Ayman Alzaatreh and M. Mansoor 2017 "The Weibull-Power Cauchy distribution : model, properties and applications". Hacettepe Journal of Mathematics and Statistics . Vol. 46 (4) , PP. 767 -789 .

(3) Lindley, D. V. 1980 “Approximate Bayesian Method ”, Trabajos de Estadistica, vol. 31, PP. 223-237 .

(4) Douglas C. M. and George C. R. 2003 “ Applied Statistics and Probability for Engineering”. Third Edition , John Wiley and Sons, Inc , PP. 1-976.

(5) Hutcheson, G. D. and Sofroniou , N. 1999 “ The Multivariate Social Scientist ”, London : Sage Publications ,PP.1228. 